\title{
Providers' and key opinion leaders' attitudes and practices regarding emergency contraception in Senegal: Key findings
}

\author{
Babacar Mane \\ Population Council \\ Martha Brady \\ Population Council \\ Saumya RamaRao \\ Population Council \\ Fatou Bintou Mbow
}

Follow this and additional works at: https://knowledgecommons.popcouncil.org/departments_sbsr-rh

Part of the Health Policy Commons, International Public Health Commons, Obstetrics and Gynecology Commons, Public Health Education and Promotion Commons, and the Women's Health Commons How does access to this work benefit you? Let us know!

\section{Recommended Citation}

Mane, Babacar, Martha Brady, Saumya RamaRao, and Fatou Bintou Mbow. 2012. "Providers' and key opinion leaders' attitudes and practices regarding emergency contraception in Senegal: Key findings," program brief. New York: Population Council. 
By Babacar Mané, Martha Brady, Saumya RamaRao, and Fatou Bintou Mbow

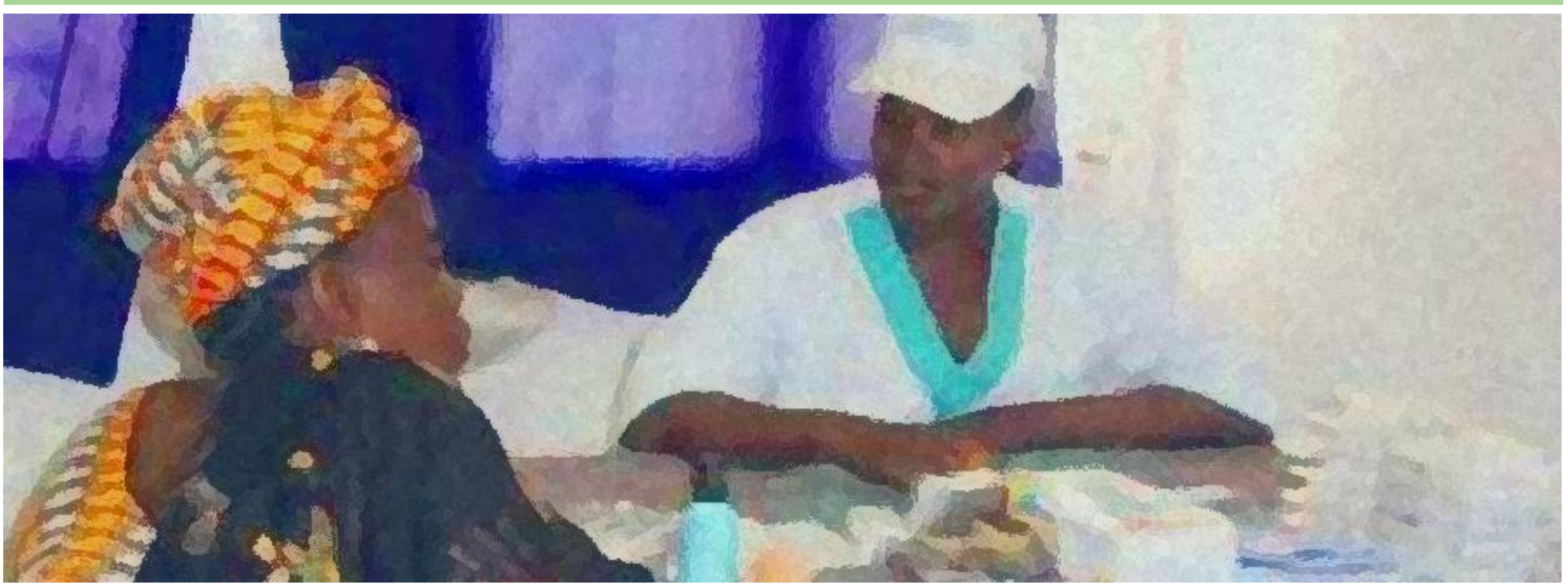

\section{BACKGROUND}

Initiatives to promote the emergency contraceptive pill (ECP) in Senegal are relatively new. The national family planning program, which is characterized by low use of modern methods and many sociocultural barriers, introduced ECP in 2000. ECP is now documented in policies, standards, and protocols relating to $\mathrm{RH} / \mathrm{FP}$ service provision and provider training programs.

Use of ECP in the public system is low (<2 percent), according to routine service statistics. In the private sector, the absence of an information system precludes monitoring ECP dispensation in pharmacies.

Access to ECP should be an important focus of the national family planning strategy, particularly given the magnitude of unintended pregnancies, sexual violence and abuse, and their consequences (unsafe abortions, infanticide). Unfortunately, lack of knowledge about ECP among providers, potential users, and policymakers is fairly widespread.

\section{OBJECTIVE}

This study examines providers' and key opinion leaders' (KOLs') knowledge, attitudes, and practices regarding ECP, and synthesizes the findings to suggest program and policy recommendations.

\section{METHODOLOGY}

The study combined qualitative and quantitative methods. A cross-sectional descriptive study of providers and KOLs was conducted in Dakar and Thies-urban areas targeted by the Urban Reproductive Health Initiative in Senegal-and in Mbour, a newly-emerging urban area. The current study is part of a larger multicountry study that includes India and Nigeria.
Thirty-four KOLs were purposively selected for interview (see Table 1 for types of KOLs).

TABLE 1 Profile of key opinion leaders (KOLs)

\begin{tabular}{lr}
\hline & $\begin{array}{r}\text { Number } \\
\text { interviewed }\end{array}$ \\
\hline Policy and program decisionmakers & 11 \\
Members of professional associations & 5 \\
NGO representatives & 3 \\
Development partners & 4 \\
Private practitioners & 1 \\
Distributors & 3 \\
Members of community-based organizations & 7 \\
Total & 34 \\
\hline
\end{tabular}

A quantitative survey was administered to a representative sample of 155 providers drawn from a range of survey delivery points, including public and private sector facilities (see Table 2 for types of facilities). Targeted providers included doctors, midwives, nurses and assis-

TABLE 2 Number and type of service delivery points from which provider sample was drawn

\begin{tabular}{lrrrr}
\hline Type of facility & Dakar & Thies & Mbour & Total \\
\hline Reference hospital & 4 & 1 & 0 & 5 \\
FP reference center & 1 & 0 & 0 & 1 \\
Health center & 14 & 0 & 1 & 15 \\
Health post & 20 & 4 & 2 & 26 \\
Adolescent counseling center & 3 & 0 & 1 & 4 \\
Private clinic & 10 & 2 & 1 & 13 \\
NGO clinic & 2 & 0 & 0 & 2 \\
Pharmacy & 30 & 4 & 2 & 36 \\
EPS 1 (level-1 hospital) & 3 & 1 & 1 & 5 \\
Academic medical center & 2 & 0 & 0 & 2 \\
Total & 89 & 12 & 8 & 109 \\
\hline
\end{tabular}


tants to nurses, pharmacists and counter staff, and service providers in youth counseling centers and private and NGO clinics.

\section{KEY FINDINGS FROM PROVIDER SURVEY}

About one-third (33 percent) of providers, particularly nurses and pharmacy staff, had not received training in family planning counseling. Moreover, training in ECP provision was not integrated into basic FP training. Nearly 4 out of 10 providers had never received specific training in ECP provision. Providers had insufficient knowledge about ECP; few were able to correctly answer questions on mode of action, when to use, and eligibility criteria. Few ECP providers gave advice or referred clients to FP services.

Analysis of providers' opinions and beliefs reveals that although many providers believed ECP is a safe product, persistent negative views remain. For example:

- Less than 45 percent of providers believed that all married women and girls should be eligible for ECP.

- Only 42 percent of trained providers were in favor of ECP being sold without prescription.

- The majority of providers supported restricting supply of ECP to conventional health facilities (hospitals, health centers, private clinics, and pharmacies); they do not favor provision of ECP in community or school settings.

- Opinions were mixed on the use of ECP among married women, unmarried adolescents, and sex workers.

- Most providers did not favor advance provision of ECP, or its use as a substitute for "regular" methods.

- Many providers believed that ECP users were likely be at risk of sexually transmitted infections, more likely to have multiple sexual relationships, and would decrease their use of nonemergency contraceptives.

\section{INSIGHTS FROM INTERVIEWS WITH KEY OPINION LEADERS}

Most KOLs consider ECP important to provide; however, they noted that reluctance to offer ECP exists among providers. One public official stated that "among providers, there are still some prejudices. They have not quite accepted this method. There is some suspicion that women may overuse it. These days, counseling is being directed toward methods such as injectables and Jadelle ${ }^{\circledR}$."

- The vast majority of KOLs were aware that ECP is a legal product available in the national program and sold in private pharmacies, but most were unaware of different ECP products.
- Some mentioned low availability of ECP, particularly in public health facilities experiencing stock shortages since 2010 due to Pregnon ${ }^{\circledR}$ expiration.

- The majority noted the high cost of ECP at private pharmacies.

- Most were in favor of integrating ECP into national guidelines for FP.

- KOLs expressed mixed views concerning provision of ECP to various population groups/targets. Some suggested ECP should be limited to certain categories of women (e.g., victims of rape and sexual assault, sex workers, women having casual sex, migrants' wives, teenagers). Others expressed that ECP should be available for all categories of women.

- The majority urged caution regarding the introduction of ECP at the community level.

- Most believed that repeat use of ECP is occurring, and that this "overuse" can have negative consequences such as exposure to STIs and substitution for "regular" contraceptives.

\section{CONCLUSION}

Both KOLs and providers recognize the importance of ECP for preventing unintended pregnancy and are supportive of the integration of ECP into national guidelines. They note, however, the need to improve accessibility and quality of provision. The vast majority of KOLs and providers believe that community awareness should be prioritized and that opportunities exist for greater acceptance of the product. Given the magnitude of sexual violence and abuse, considerable interest exists in devising strategies to protect the sexual and reproductive health and welfare of adolescents and young women, including through enhancing the provision of ECP. Such strategies might include:

1. Creating public awareness campaigns and activities to inform communities about ECP.

2. Training all cadres of providers.

3. Strengthening the logistics management system to ensure the availability of the product.

4. Improving and monitoring pharmacy provision of ECP.

5. Conducting policy advocacy activities.

6. Developing an agenda for further research tailored to ECP knowledge, attitudes, and provision in particular contexts.

\footnotetext{
The Population Council confronts critical health and development issues-from stopping the spread of HIV to improving reproductive health and ensuring that young

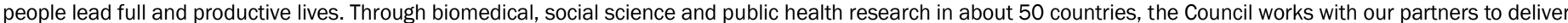

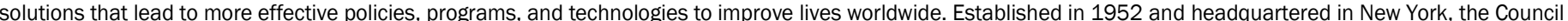
is a nongovernmental, nonprofit organization with an international board of trustees.
}

www.popcouncil.org 